\title{
MACC1 decreases the chemosensitivity of gastric cancer cells to oxaliplatin by regulating FASN expression
}

\author{
JIANGMAN DUAN $^{1,4^{*}}$, LISHAN CHEN $^{2 *}$, MINYU ZHOU $^{1}$, JINGWEN ZHANG $^{1}$, LI SUN $^{1}$, \\ NA HUANG ${ }^{1}$, JIANPING BIN ${ }^{3}$, YULIN LIAO ${ }^{3}$ and WANGJUN LIAO ${ }^{1}$ \\ ${ }^{1}$ Department of Oncology, Nanfang Hospital, Southern Medical University, Guangzhou 510515; ${ }^{2}$ Huiqiao Medical Center, \\ Nanfang Hospital, Southern Medical University, Guangzhou 510515; ${ }^{3}$ Department of Cardiology, Nanfang Hospital, \\ Southern Medical University, Guangzhou 510515; ${ }^{4}$ Department of Oncology, \\ Shenzhen Nanshan Hospital, Shenzhen 518052, P.R. China
}

Received July 31, 2016; Accepted November 24, 2016

DOI: 10.3892/or.2017.5519

\begin{abstract}
The effect of chemotherapeutic agents is limited as a result of drug resistance, which demands prompt solutions provided by clinical studies. To date, the underlying mechanisms of chemotherapy resistance are relatively unknown. Metastasis-associated in colon cancer 1 (MACC1) is an oncogene associated with the progression and prognosis of gastric cancer (GC). Bioinformatic analysis revealed that MACC1 is positively associated with fatty acid synthase (FASN), a major enzyme of lipogenesis, and drives chemoresistance to oxaliplatin in GC. Similar findings were demonstrated in two GC cell lines (BGC-823 and MKN-28) with MACC1 ectopic expression. We next employed FASN inhibitor $\mathrm{C} 75$ or siFASN (small interfering RNA targeted to FASN) to block endogenous fatty acid metabolism and it was revealed that cell proliferation and chemoresistance to oxaliplatin induced by MACC1 upregulation were attenuated by FASN blockade to various extents. Conclusively, these outcomes highlight a novel role of MACC1 in GC cell lipogenesis, and suggest that MACC1 may be an attractive target to decrease oxaliplatin resistance in GC.
\end{abstract}

\section{Introduction}

State-of-the-art advanced gastric cancer (GC) treatment mainly involves chemotherapy and targeted therapy. In most cases, however, intrinsic or acquired chemoresistance occurs and results in a 5-year survival rate of less than $10 \%$ in patients diagnosed with advanced disease (1). Therefore, chemoresistance is the main cause of the poor prognosis in

Correspondence to: Professor Wangjun Liao, Department of Oncology, Nanfang Hospital, Southern Medical University, 1838 North Guangzhou Avenue, Guangzhou 510515, P.R. China E-mail: nfyyliaowj@163.com

*Contributed equally

Key words: MACC1, FASN, oxaliplatin, chemosensitivity, gastric cancer
GC. As a third-generation platinum derivative, oxaliplatin has been successfully applied in treating gastrointestinal tumors. Although, responsiveness to oxaliplatin is initially high, patients ultimately develop oxaliplatin resistance (2). Nevertheless, the exact mechanisms involved in oxaliplatin resistance in GC have not been elucidated. Thus, it is essential to identify novel therapeutic strategies for decreasing the resistance of GC to oxaliplatin-based chemotherapy.

Metastasis-associated in colon cancer 1 (MACC1), as the name suggests, was originally identified as a colon cancer oncogene promoting metastasis (3). Our previous studies demonstrated that MACC1 is involved in the process of gastric malignancy, enhances GC cell proliferation and invasiveness, and is associated with abnormal glucose metabolism $(4,5)$. MACC1 has been confirmed to be a metabolic oncogene, however, little has been reported concerning MACC1 and oxaliplatin resistance in GC. Emerging evidence suggests that altered metabolism in cancer cells is fundamentally involved in the development of drug resistance (6-8). The targeting of key metabolic enzymes which sustain these cancerous metabolic adaptations bears great promise for improving treatment efficacy in patients with metastatic diseases $(7,9)$. Hence, we hypothesized that metabolism bridges MACC1 and oxaliplatin resistance. Various studies have found that the key enzymes of lipid metabolism are involved in drug resistance (10-12). Furthermore, through the bioinformatic analysis from cBio Cancer Genomics Portal (http://www.cbioportal.org), we explored the relationships between MACC1 and key enzymes in lipid metabolism and found that the expression of MACC1 is positively associated with fatty acid synthase (FASN) in GC.

The multi-enzyme protein FASN is a key enzyme in lipid metabolism, whose expression has great potential in helping to understand disorders of lipid metabolism, which is of great importance in GC progression $(13,14)$. In previous studies, we found that FASN was highly expressed in GC tissues and that the overexpression of FASN was associated with the poor prognosis of GC patients (15-17). More importantly, FASN is reported to be a druggable gene and has contributed to platinum-resistance in several types of tumors (18-20). However, the probable underlying mechanisms remain unknown. Therefore, we hypothesized that FASN inhibition contributes 
to oxaliplatin resistance in GC and we further explored the probable mechanisms involved.

Hence, in the present study, we tried to determine the correlation of MACC1, FASN and oxaliplatin resistance in GC. For this purpose, IHC, in vivo and in vitro studies were conducted to clarify the detailed relationship between MACC1 and FASN. Moreover, drug-sensitivity experiments were also performed to ascertain that MACC1 decreased the chemosensitivity of GC cells to oxaliplatin through the regulation of FASN expression.

\section{Materials and methods}

Patients and tissue specimens. The present study was approved by the Ethics Review Board of Nanfang Hospital, Southern Medical University (Guangzhou, China). The present study was conducted on tissue specimens from 167 patients who had been histologically diagnosed with GC at Nanfang Hospital from 2005 to 2012 and tumor stage was defined according to the AJCC Cancer Staging Manual (7th edition, 2010). Among them, 131 stage I-III patients received radical resection $(19,49$ and 63 for stages I, II and III, respectively), and 36 stage IV patients (with metastasis in distant organs) underwent palliative surgery and/or chemotherapy. Postoperative follow-up ranging from 0.5 to 80.0 months was obtained for all patients. Twelve paired tumors and corresponding normal gastric mucosa were rapidly removed at surgery and stored at $-80^{\circ} \mathrm{C}$ in a freezer for RNA or protein extraction.

Cell culture and transfection assays. Human gastric adenocarcinoma poorly differentiated BGC-823 and well-differentiated MKN-28 cell lines were obtained from Foleibao Biotechnology Development Co. (Shanghai, China). Stably-transfected MACC1-overexpressing and silenced cell lines were established and cultured as described in our previous published study (16). Transient transfection of FASN-specific small interfering RNAs (siRNAs) (RiboBio Co., Guangzhou, China) was performed using Lipofectamine 2000 (Invitrogen, Carlsbad, CA, USA) on BGC-823 and MKN-28 cell lines. A non-specific scrambled siRNA (Ctrl) was used as a negative control. The silencing sequences of FASN-siRNAs are listed in Table I.

MTT and EdU assays. The effects of siFASN and FASN inhibitor $\mathrm{C} 75$ on cell proliferation were determined by 3-(4,5-dimethylthiazol-2-yl)-2,5-diphenyltetrazolium bromide (MTT) and EdU incorporation assays. Briefly, GC cells were seeded in a 96-well plate 1 day before treatment with $10 \mu \mathrm{g} / \mathrm{ml}$ C75 for $24 \mathrm{~h}$ or $50 \mathrm{nM}$ siFASN for $48 \mathrm{~h}$. After incubation, MTT solution $[5 \mathrm{mg} / \mathrm{ml}$ in phosphate-buffered saline (PBS)] was added to each well in an amount equal to $10 \%$ of the culture medium volume. Cells were then incubated for $4 \mathrm{~h}$ at $37^{\circ} \mathrm{C}$, and the absorbance was evaluated at $570 \mathrm{~nm}$ using a microplate spectrophotometer (Thermo Scientific, Franklin, MA, USA). Each assay was performed in triplicate. All experiments were repeated three times. In addition, EdU incorporation assay was conducted using the EdU assay kit (RiboBio Co.) according to the manufacturer's instructions. Cells were incubated with $50 \mathrm{nM}$ of EdU for an additional $2 \mathrm{~h}$ at $37^{\circ} \mathrm{C}$ after treatment. Cells were fixed with $4 \%$ formaldehyde for $15 \mathrm{~min}$ at room
Table I. FASN-siRNA silencing sequences.

\begin{tabular}{lll}
\hline siRNA1 & F & GGACCUGUCUAGGUUUGAUdTdT \\
& R & dTdTCCUGGACAGAUCCAAACUA \\
siRNA2 & F & UGGAGCGUAUCUGUGAGAAdTdT \\
& R & dTdTACCUCGCAUAGACACUCUU \\
siRNA3 & F & CCUGCGUGGCCUUUGAAAUdTdT \\
& R & dTdTGGACGCACCGGAAACUUUA
\end{tabular}

FASN, fatty acid synthase; siRNA, small interfering RNA; F, forward; R, reverse.

Table II. Primer sequences for quantitative real-time PCR.

\begin{tabular}{llcc}
\hline Gene name & \multicolumn{2}{c}{ Primer sequences (5' to 3') } & $\begin{array}{c}\text { Primer } \\
\text { length } \\
\text { (bps) }\end{array}$ \\
\hline MACC1 & F & GGCTGTGATGCTACGAGATA & 20 \\
& R & ACACCAGGACAATGCCTACT & 20 \\
FASN & F & CGACAGCACCAGCTTCGCCA & 20 \\
& R & CACGCTGGCCTGCAGCTTCT & 20 \\
ACC & F & TCAAACTGCAGGTATCCCAAC & 20 \\
& R ATTTCCTGCCAGTCCACAC & 20 \\
ACLY & F & GAAGGGAGTGACCATCATCG & 20 \\
& R & TTAAAGCACCCAGGCTTGAT & 20 \\
GAPDH & F & ACTTCAACAGCGACACCCACTC & 22 \\
& R & TACCAGGAAATGAGCTTGACAAAG & 24
\end{tabular}

MACC1, metastasis-associated in colon cancer 1; FASN, fatty acid synthase; ACC, Acetyl-CoA carboxylase; ACLY, ATP-citrate lyase; $\mathrm{F}$, forward; $\mathrm{R}$, reverse.

temperature and treated with $0.5 \%$ Triton X-100 for $20 \mathrm{~min}$ at room temperature to permeate cell membranes. After being washed with PBS three times, the cells were incubated with $1 \mathrm{X}$ Apollo reaction cocktail (100 $\mu \mathrm{l} /$ well) for $30 \mathrm{~min}$. DNA was stained with $10 \mu \mathrm{g} / \mathrm{ml}$ of Hoechst 33342 stain $(100 \mu \mathrm{l} /$ well) for $20 \mathrm{~min}$ and images were visualized with fluorescence microscopy. Five fields of view were randomly selected for each sample. EdU-positive cells were stained with red dye, and the relative proliferation-positive ratios were calculated from the average cell count of the five visual fields.

Quantitative real-time PCR. GC tissue specimens for quantitative real-time PCR (qRT-PCR) analysis were obtained from 20 patients, who underwent surgery from November-December 2011. None of these patients had received chemotherapy prior to surgery. Primer sequences involved in the present study are summarized in Table II. A TRIzol kit (Life Science, Carlsbad, CA, USA) for total RNA extraction and a reverse transcriptase kit (Roche, Penzberg, Germany) for cDNA synthesis were used according to the manufacturer's protocols. qRT-PCR was performed using SYBR-Green I Master kit (Roche) on a LightCycler 480 system. 
Western blotting. Cell lysates were first loaded onto SDS-PAGE for electrophoresis, then, transferred to polyvinylidene difluoride membranes. The membranes were blocked with $5 \%$ non-fat milk for $1 \mathrm{~h}$, and probed with anti-FASN (Cell Signaling Technology, Danvers, MA, USA) and anti-GAPDH (Novus, St. Charles, MO, USA) antibodies at $4^{\circ} \mathrm{C}$ overnight. Membranes were washed in Tris-Tween buffer saline, and incubated with horseradish peroxide-conjugated secondary antibodies (Kirkegaard \& Perry Laboratories, Gaithersburg, MD, USA) at room temperature for $1 \mathrm{~h}$. The enhanced chemiluminescence method with a western blotting detection system (Kodak Digital Science, Rochester, NY, USA) was used. Image software Quantity One v4.6.2 was used for the intuitionistic and quantitative analysis.

Immunohistochemistry. Immunohistochemical staining was carried out according to the Dako EnVision System (Dako, Glostrup, Denmark). The sections were deparaffinized, rehydrated in serially graded ethanol, and heated $5 \mathrm{~min}$ in citric buffer ( $\mathrm{pH}$ 6.0) once for antigen retrieval. They were then washed with distilled water, blocked with $3 \%$ hydrogen peroxide and incubated overnight with the primary antibodies including MACC1 (Abcam, Cambridge, UK), FASN (Cell Signaling Technology, Inc., Danvers, MA, USA) at $4^{\circ} \mathrm{C}$. After being washed with a $0.01 \mathrm{M}$ concentration of PBS, a secondary antibody solution (biotinylated antibody solution; Dako) was added for $1 \mathrm{~h}$ at room temperature. Subsequently, the sections were washed, stained with 3,3'-diaminobenzidine (DAB) chromogen (Dako) and counterstained with hematoxylin (17). Target protein expression levels in tumor tissues were scored using a semi-quantitative method as previously described with modifications.

Animals and establishment of subcutaneous tumor models. Four-week-old male athymic BALB/c nude mice were obtained from Sun Yat-Sen University (Guangzhou, China). All animal experiments followed the ethical guidelines formulated by the Institute of Experimental Animal of Southern Medical University. Mice were housed in specific pathogen-free conditions with standard laboratory food for mice and water. BGC823 GC cells $\left(1 \times 10^{7}\right)$ in $0.3 \mathrm{ml}$ of PBS were subcutaneously injected into the left and right posterior flank regions of each mouse, and tumor growth was assessed every three days using calipers. Mice were randomly chosen and assigned to two groups (five mice/group) according to different expression levels of MACC1 (oxMACC1 and the control group, shMACC1 and the control group). After 28 days, mice were sacrificed and the tumor volumes were determined in accordance with the formula: $\mathrm{V}=\mathrm{a} \times \mathrm{b} \times(\mathrm{a}+\mathrm{b}) / 2$, where ' $\mathrm{a}$ ' is length and ' $\mathrm{b}$ ' is width of the tumor, respectively.

Statistical analysis. Statistical calculations were performed using SPSS 20.0 software (version 20.0; SPSS, Inc., Chicago, IL, USA). Survival analysis was performed from the date of surgery to the time of diagnosis of recurrence or death using the Kaplan-Meier method. The relationship between MACC1 and FASN was analyzed by statistical linear regression. The significant differences were calculated using Student's t-test for continuous variables. Results of the MTT assay were analyzed by repeated measures. The quantitative data are expressed as
Table III. Correlation between MACC1 and three key enzymes of lipogenesis.

\begin{tabular}{lcc}
\hline Gene symbol & Pearson score & Spearman score \\
\hline ACC & - & - \\
ACLY & 0.21 & 0.32 \\
FASN & 0.09 & 0.21 \\
\hline
\end{tabular}

MACC1, metastasis-associated in colon cancer 1; ACC, Acetyl-CoA carboxylase; ACLY, ATP-citrate lyase; FASN, fatty acid synthase.

the mean of three independent experiments. P-values $<0.05$ were defined as statistically significant.

\section{Results}

MACC1 and FASN are positively correlated and responsible for the poor prognoses in GC patients. We demonstrated that MACC1 was overexpressed in GC and was related to glycometabolism in previous studies $(4,5)$. In the present study, we focused on the relevance between MACC1 and tumor lipid metabolism. We commenced a systematic research on the relationship between MACC1 and three key enzymes in lipid metabolism, namely Acetyl-CoA carboxylase (ACC), ATP-citrate lysase (ACLY) and FASN. First, bioinformatics website http://www.cbioportal.org was employed to explore whether there was a correlation between MACC1 and these three key enzymes. A marked correlation was found between MACC1, ACLY and FASN in GC, with the correlation coefficients listed in Table III.

To confirm the reliability of the data, we collected GC tissues from 9 clinical cases and the total RNA was extracted. The gene expression of MACC1 and these three key enzymes of lipogenesis were synchronously analyzed. As indicated in Fig. 1A, the expression of MACC1 and FASN was significantly correlated $(\mathrm{r}=0.831, \mathrm{p}=0.003)$, while no significant correlation was found between MACC1 and ACC ( $\mathrm{r}=-0.149$, $\mathrm{p}=0.351)$ or ACLY ( $\mathrm{r}=-0.414, \mathrm{p}=0.134)$. Based on these results, we suggested that MACC1 may be correlated with FASN, one of the key enzymes of lipogenesis. Next, we conducted qRT-PCR to assess the mRNA levels of these three enzymes in the MACC1 stably-transfected GC cell lines. According to our results, overexpression of MACC1 (oxMACC1) stimulated FASN gene expression efficiently compared to its negative control (vector), while knockdown of MACC1 (shMACC1) reversed this tendency when compared to its control cell line (scramble) (Fig. 1B). Based on the results obtained, we considered that among these three key enzymes involved in the process of de novo fatty acid synthesis, FASN was the main enzyme that had a positive correlation with MACC1 in GC.

Statistical analysis was then conducted based on the immunohistochemical staining of the protein levels of MACC1 and FASN in the 167 cases of GC patients. Linear-regression analysis indicated a marked correlation between MACC1 and FASN protein expression ( $\mathrm{p}<0.001$; Fig. 1C). In addition, Kaplan-Meier survival analysis revealed a shorter diseasefree survival (DFS) for stage I-III and overall survival (OS) 

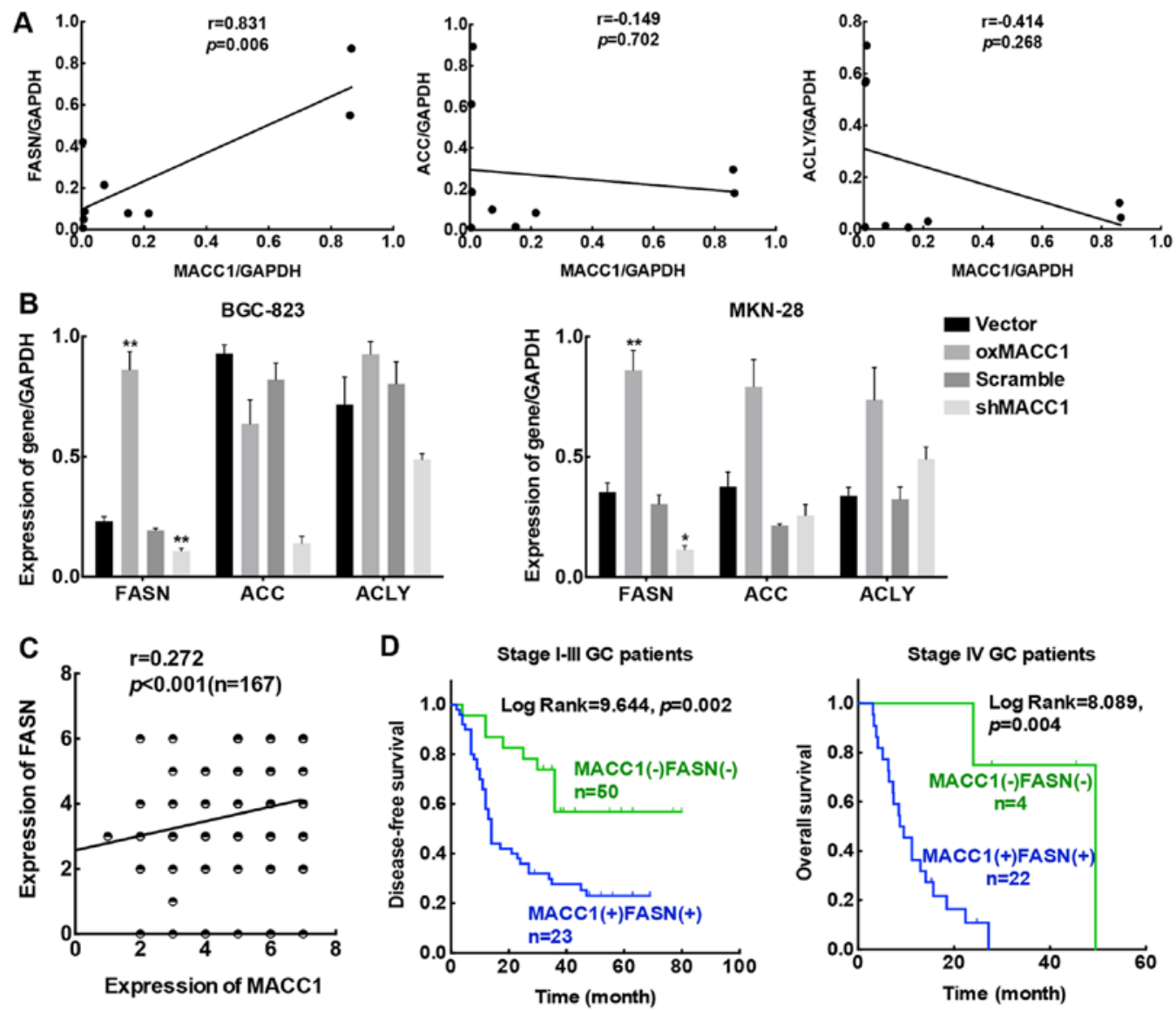

Figure 1. MACC1 and FASN are positively correlated and responsible for poor prognoses in GC. (A) The correlation between MACC1 and three key enzymes of lipogenesis, ACC, ACLY and FASN in 9 clinical specimens. (B) Gene expression levels of ACC, ACLY and FASN in MACC1 stably-transfected GC cells. Data are the results obtained from triple independent experiments and are expressed as the mean \pm SD. (C) Linear regression analysis of MACC1 and FASN protein expression in 167 GC patient tissues. (D) Kaplan-Meier survival plots of DFS for stage I-III and OS for stage IV patients according to MACC1 and FASN expression; " $\mathrm{p}<0.05,{ }^{* * *} \mathrm{p}<0.01$. MACC1, metastasis-associated in colon cancer 1; FASN, fatty acid synthase; GC, gastric cancer; ACC, acetyl-CoA carboxylase; ACLY, ATP-citrate lysase; DFS, disease-free survival; OS, overall survival.

for stage IV patients with high expression of both MACC1 and FASN than those with low expression of MACC1 and FASN (Fig. 1D). Thus, we ascertained that the expression of MACC1 was positively correlated with FASN in GC tissues. Moreover, co-expression of MACC1 and FASN predicted a poor prognosis for GC patients. These findings highly suggest that MACC1 contributes to GC progression partly through abnormal tumor lipid metabolism.

MACC1 positively regulates FASN expression in vitro and in vivo. According to the clinical data, we concluded that the expression of MACC1 and FASN were closely correlated at both mRNA and protein levels. Therefore, we next investigated their relationship both in vitro and in vivo. To begin with, we extracted the total protein of two MACC1 stably-transfected GC cell lines (vector, oxMACC1, scramble and shMACC1 for BGC-823 and MKN-28 cell lines). Western blotting results revealed that the protein levels of FASN were significantly increased in the oxMACC1 group and concurrently downregulated after silencing MACC1 (Fig. 2A).

Considering that after stable-transfection of MACC1, FASN protein expression was altered, we next detected whether the enzyme activity of FASN was concomitantly changed. We found that MACC1 overexpression markedly enhanced the activity of FASN, while silencing of MACC1 significantly downregulated the enzyme activity, respectively (Fig. 2B). These results demonstrated that interference with MACC1 could not only alter the expression of FASN in both gene and protein levels, but also modulate its enzyme activity.

Finally, we established xenograft gastric tumor models by subcutaneously injecting MACC1 stably-transfected GC BGC-823 cells into nude mice. After four weeks when xenograft tumors formed, the local tumor and lung metastatic lesions were both utilized for IHC staining. We found that in both xenograft and metastatic tissues, tumors from the groups injected with the oxMACC1 cells exhibited high expression of FASN, while the tumors derived from the shMACC1 cells exhibited significantly low expression of FASN, compared to the control groups, respectively (Fig. 2C). We demonstrated that MACC1 upregulated FASN expression in vitro and in vivo at both the gene and protein levels.

FASN inhibition attenuates proliferation in GC cell lines without MACCl variation. The foregoing experiments indicated that MACC1-overexpressing BGC-823 and MKN-28 cells had upregulated FASN expression at both the mRNA and protein levels. Therefore, we introduced a synthesized FASN inhibitor (C75) and siRNA separately to suppress 
A

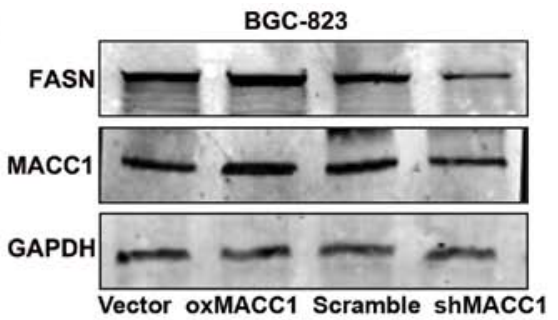

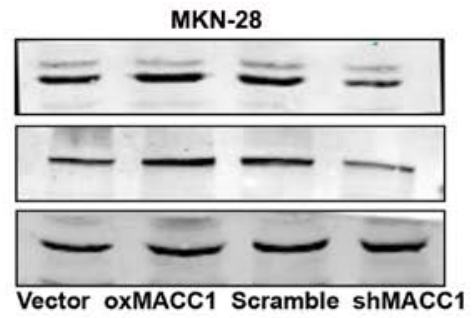

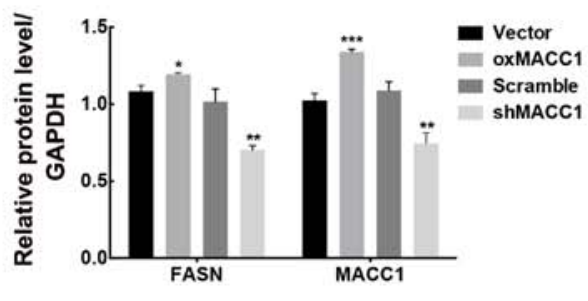

B
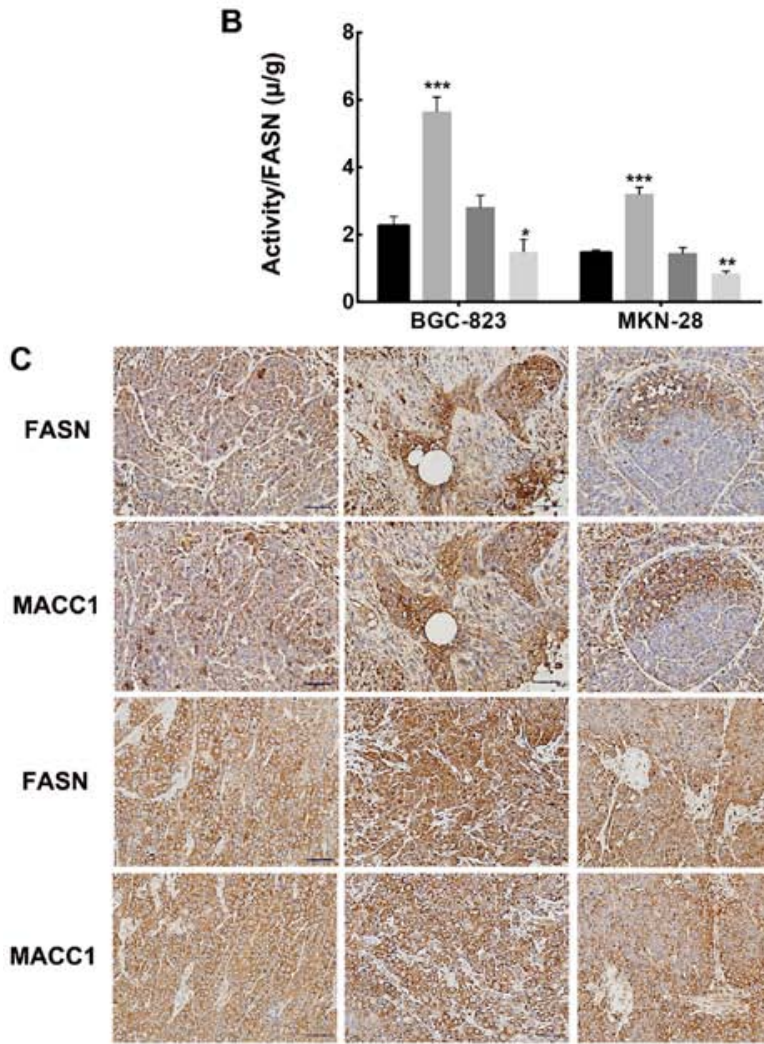

Vector

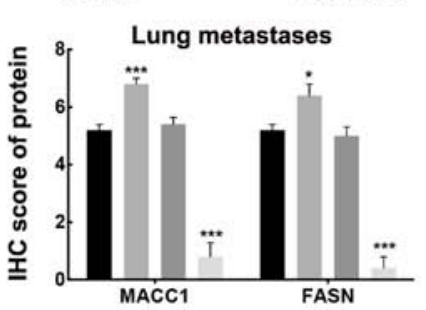

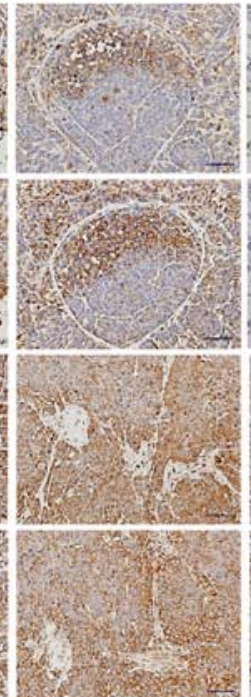

Scramble
Vector

oxMACC1

Scramble

shMACC1
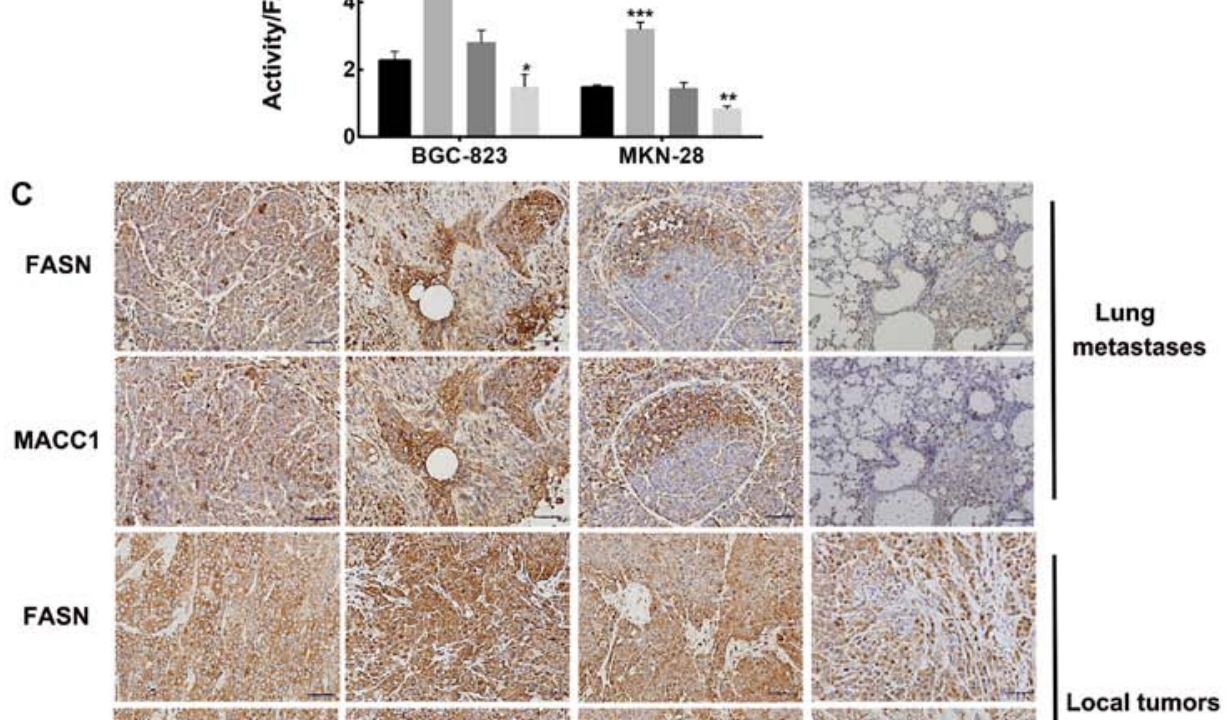

Subcutaneous tumors

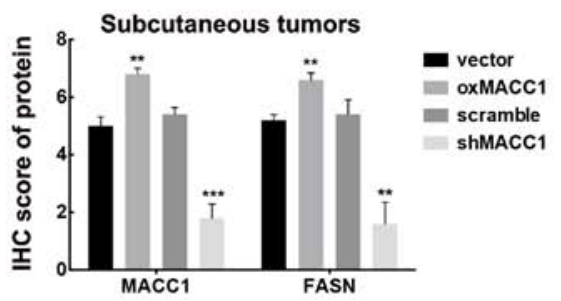

Figure 2. MACC1 positively regulates FASN expression in vitro and in vivo. (A) Protein level alterations of FASN in MACC1 stably-transfected GC cell lines (BGC-823 and MKN-28). Data are expressed as the mean \pm SD from three replicate experiments. (B) FASN enzyme activity evaluation in MACC1 stably-transfected GC cell lines (BGC-823 and MKN-28). Data are expressed as the mean \pm SD from three replicate assays. (C) FASN protein levels in in vivo mouse xenograft and lung metastasis tumor models established from MACC1 stably-transfected GC cells $(\mathrm{BGC}-823) ;{ }^{*} \mathrm{p}<0.05,{ }^{* *} \mathrm{p}<0.01,{ }^{* * *} \mathrm{p}<0.001 . \mathrm{MACC} 1$, metastasis-associated in colon cancer 1; FASN, fatty acid synthase; GC, gastric cancer.

the expression of FASN in the MACC1 stably-transfected cell lines. First of all, the optimal concentration of $\mathrm{C} 75$ in the GC cell lines was determined using an MTT assay. The results indicated a marked decrease in cell viability as C75 concentration increased (Fig. 3A). Next, C75 or the control blank solvent were added into the cell culture medium of wildtype GC cells for a 24-h incubation, after which cells were collected for qRT-PCR and western blotting. In both wild-type BGC-823 and MKN-28 cells, C75 treatment generated a significant decrease in FASN mRNA levels compared to the 

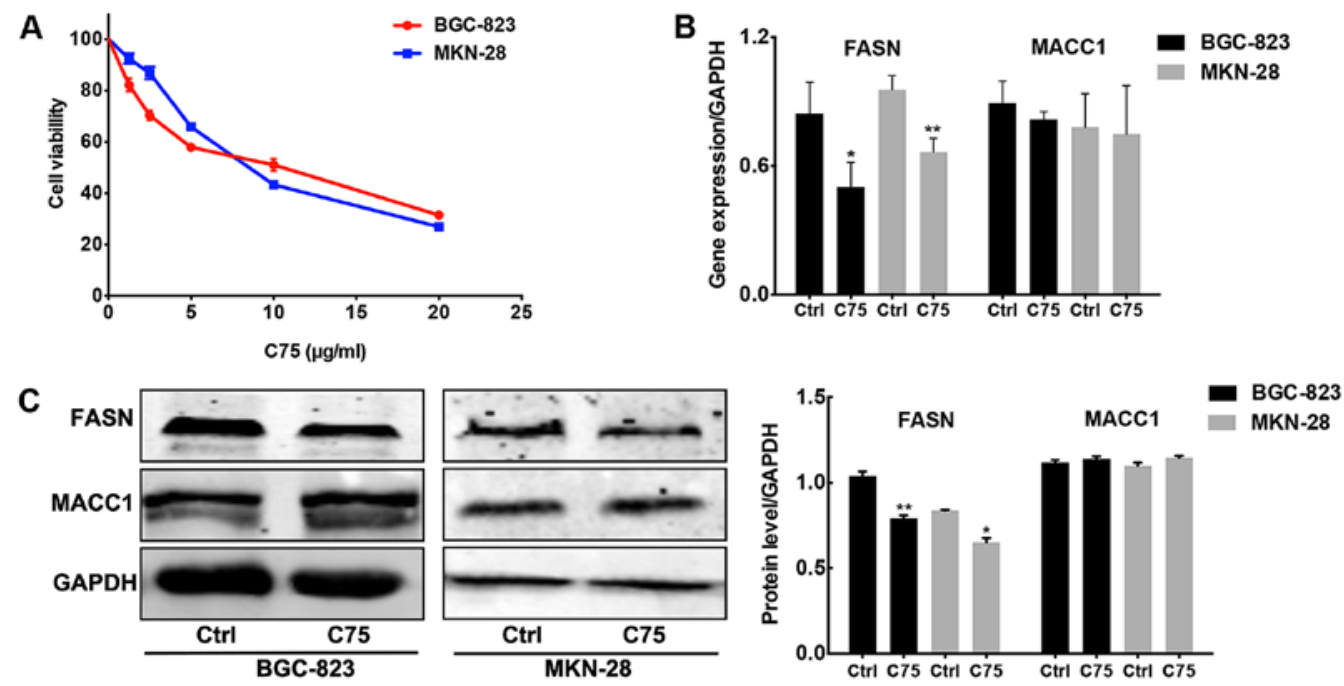

D
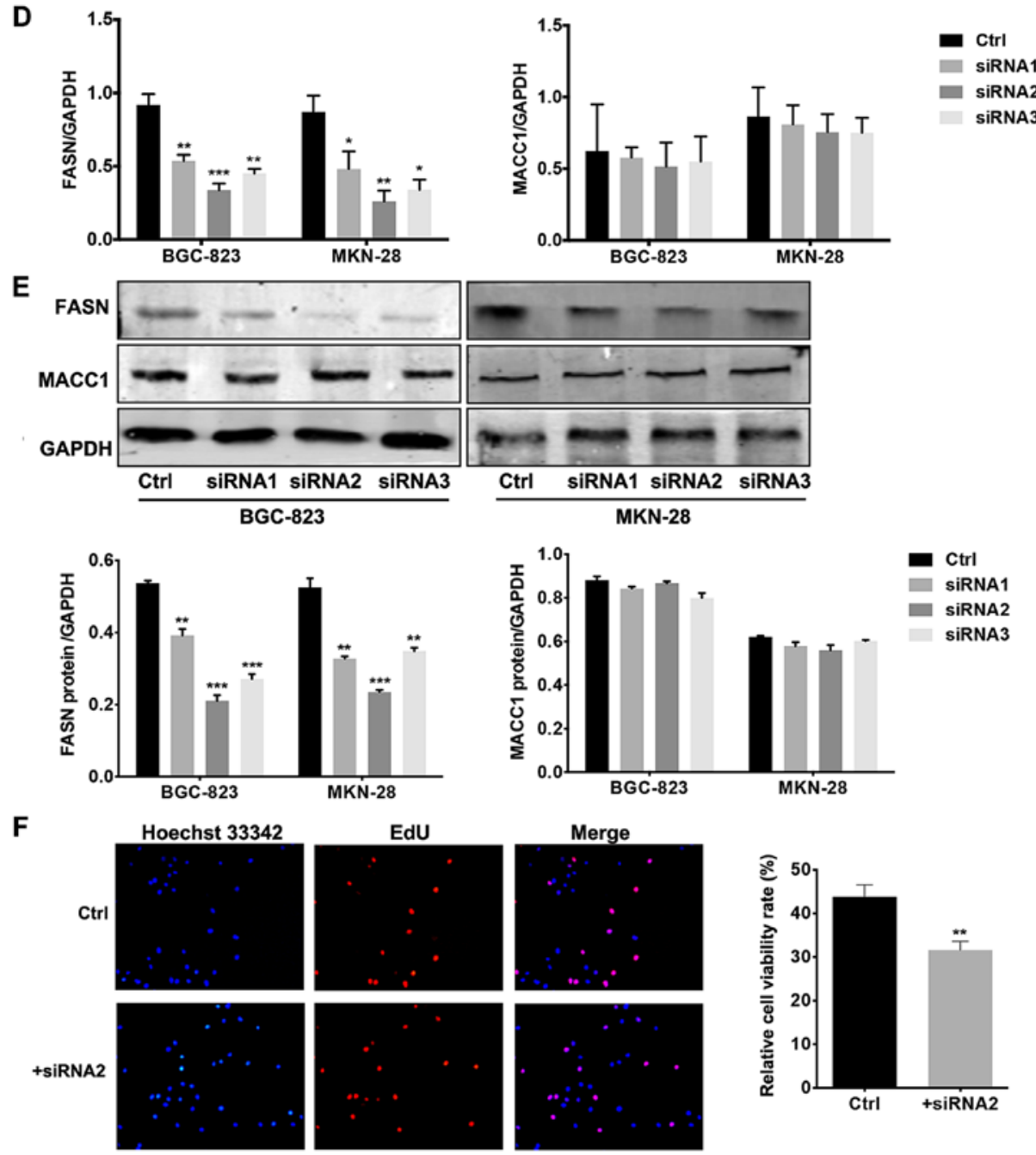

Figure 3. FASN inhibition attenuates proliferation in GC cell lines without MACC1 variation. (A) Cell viability curves of BGC-823 and MKN-28 cells treated with C75, the FASN inhibitor. (B) FASN and MACC1 gene expression levels of the control or the C75-treatment groups. (C) Western blotting for protein level detection of FASN and MACC1 in the control or the C75-treatment group. (D) FASN and MACC1 mRNA level alterations with FASN-siRNA or control-siRNA transfection. Data are results obtained from triple independent experiments and are expressed as the mean \pm SD. (E) Western blotting for protein level observation of FASN and MACC1 with FASN-siRNA transfection. (F) EdU assay for FASN-siRNA2-transfected BGC-823 cells and cell proliferation rates were quantified from five random microscopic fields; ${ }^{*} \mathrm{p}<0.05,{ }^{* *} \mathrm{p}<0.01,{ }^{* * * *} \mathrm{p}<0.001$. FASN, fatty acid synthase; GC, gastric cancer; MACC1, metastasis-associated in colon cancer 1.

control group ( $\mathrm{p}<0.05$ for BGC-823; $\mathrm{p}<0.01$ for MKN-28). The inhibitory rate for FASN was 58.8 and $69.8 \%$, respectively, but no difference in MACC1 mRNA expression was observed in both groups ( $p>0.05$; Fig. 3B). Similarly, FASN expression was 

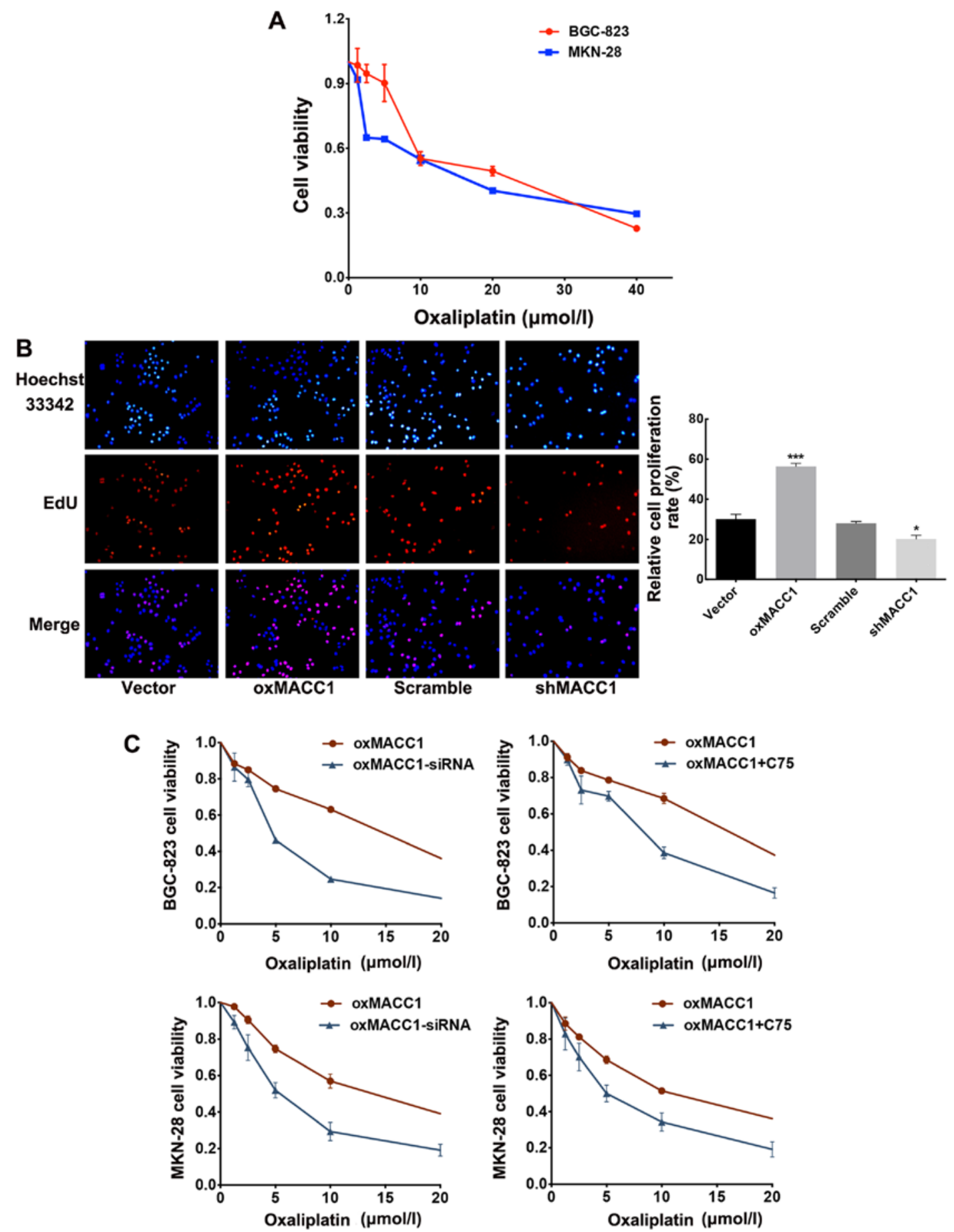

Figure 4. Overexpressing MACC1 attenuates chemosensitivity, while silencing FASN restores drug sensitivity to oxaliplatin in GC. (A) Cell viability curve of BGC-823 and MKN-28 cells treated with oxaliplatin at different concentrations. (B) EdU assay for MACC1 stably-transfected BGC-823 cells with oxaliplatin incubation $(10 \mu \mathrm{mol} / 1)$ for $24 \mathrm{~h}$. The results were expressed as the mean \pm SD calculated from five random microscopic fields. (C) MACC1-overexpressing BGC-823 and MKN-28 cells were perturbed with either C75 or FASN-siRNA before oxaliplatin treatment $(0-20 \mu$ mol/l). Cell viability was calculated as the mean $\pm \mathrm{SD}$ of three independent experiments; ${ }^{*} \mathrm{p}<0.05,{ }^{* * *} \mathrm{p}<0.001$. FASN, fatty acid synthase; GC, gastric cancer; MACC1, metastasis-associated in colon cancer 1 .

significantly suppressed by $\mathrm{C} 75$ at the protein level in GC cells, while expression of MACC1 was not significantly decreased by the FASN inhibitor (Fig. 3C). These results suggested that MACC1, working as the upstream molecule, regulated the expression of FASN.

The aforementioned results indicated that exogenous blockade of FASN had no influence on MACC1 expression. For a more thorough and comprehensive elucidation, siRNA was introduced. FASN-siRNA was transfected into GC cells using Lipofectamine 2000 to suppress FASN expression endogenously. BGC-823 and MKN-28 cells were both Transit-transfected with FASN-siRNA1, siRNA2, siRNA3 and control siRNA at the optimal concentration following the protocol instructions. After a 48-h transfection, cells were collected for qRT-PCR and western blotting to analyze the perturbation efficiency of FASN and the expression 
status of both FASN and MACC1. The expression of FASN was significantly downregulated by siRNAs in the poorly differentiated BGC-823 cells and the welldifferentiated MKN-28 cells at both the mRNA and protein levels (Fig. 3D and E). We compared the effect of the three FASN-siRNAs with different sequences on FASN blockade at both the gene and protein levels in BGC-823 and MKN-28 cells and selected the most effective sequence FASN-siRNA2 for follow-up function experiments.

In addition, we further explored the detailed function of FASN in GC progression. Endogenous FASN in poorly differentiated BGC-823 cells was blocked through FASNsiRNA transfection. An EdU proliferation assay was conducted to explore cell proliferation activity. As expected, the cellular fluorescent images clearly revealed decreased cell proliferation with FASN inhibition. The quantitative analysis indicated a significant difference $(\mathrm{p}<0.01$; Fig. 3F). These results provide evidence that FASN inhibition attenuates proliferation in GC cell lines without MACC1 variation.

Overexpression of MACC1 attenuates chemosensitivity, while silencing of FASN restores drug sensitivity to oxaliplatin in $G C$. As numerous studies have confirmed that MACC1 and FASN are both druggable targets, which contribute to platinum-resistance in several tumors $(10,24,25)$, the present experiment explored whether MACC1 and FASN affect the chemosensitivity of GC cells to oxaliplatin. We first studied the inhibitory effect of oxaliplatin on wild-type GC cells. A gradient of six concentrations $(0,1.25,2.5,5,10$ and $20 \mu \mathrm{mol} / \mathrm{l})$ of oxaliplatin was employed separately to stimulate BGC-823 and MKN-28 cells for $24 \mathrm{~h}$ and the resulting cell viability was assessed with an MTT assay (Fig. 4A). We noted that in both cell lines, cell viability was decreased as drug dosage increased. A high concentration of oxaliplatin had a more obvious inhibitory effect on cell proliferation and cytotoxicity increased as well. Therefore, we calculated the $\mathrm{ID}_{50}$ value of oxaliplatin for both cell lines and decided that the optimal concentration for subsequent experiments was $10 \mu \mathrm{mol} / 1$.

Ensuingly, we employed EdU cell proliferation assay on MACC1 stably-transfected cells after oxaliplatin incubation $(10 \mu \mathrm{mol} / \mathrm{l})$ for $24 \mathrm{~h}$. Cellular fluorescence intuitively revealed higher proliferation activity in the MACC1-overexpressing cells than that noted in the control vector group $(\mathrm{p}<0.001$; Fig. 4B); conversely, shMACC1 and its control scramble cells demonstrated a reverse tendency, which exhibited decreased proliferation activity in the shMACC1 cells compared with the scramble group $(\mathrm{p}<0.005)$.

The aforementioned results suggested that MACC1 suppressed the drug-sensitivity of GC cells to oxaliplatin. We further explored the probable underlying mechanism in the following experiment. With this in mind, we assumed that MACC1 regulated the drug sensitivity of GC cells to oxaliplatin through the modulation of the key enzyme of lipogenesis FASN. To confirm our conjecture, we treated MACC1-overexpressing cells with either C75 or FASN-siRNA for $24 \mathrm{~h}$ to suppress the expression of FASN. Then, an MTT assay was performed to assess the inhibition efficacy of oxaliplatin on cell proliferation. We found that after inhibition of FASN by exogenous artificially synthesized compound C75, oxaliplatin slowed cell proliferation more efficiently than the untreated control group. Furthermore, we introduced FASN-siRNA in the MACC1-overexpressing GC cells to block endogenous FASN expression at the genetic level. The same result was obtained which indicated an enhanced sensitivity to oxaliplatin and a decreased proliferation ability of MACC1-overexpressing cells after FASN blockade (Fig. 4C). In general, the aforementioned experiments ascertained our previous hypothesis that upregulation of FASN by MACC1 attenuated drug sensitivity of GC cells to oxaliplatin.

\section{Discussion}

Tumor cells are commonly characterized by high glucose uptake and consumption ascribed to the low efficiency of aerobic glycolysis (21), and lipometabolism is an additional pathway which is crucial in sustainable energy supplementation for its rapid proliferation. Within the organism, a set of precise lipometabolic networks regulate the process of lipid biosynthesis and lipolysis (22), which ensure the normal structure and function of cells. Multiple studies have shown that lipometabolic disorders are associated with the occurrence and development of many diseases including tumors (23). Our preliminary study revealed that MACC1 is a critical protein related to GC cell metabolism, which mediates GC glucose metabolism, neovascularization and lymph angiogenesis $(4,24)$. However, to date there has been no investigation on the role of MACC1 in GC cell lipometabolism. Using bioinformatic analysis from cBio Cancer Genomics Portal (http://www.cbioportal.org), we found that the expression of MACC1 is closely associated with key enzymes of lipometabolism (ACLY and FASN) in GC, highly suggesting that MACC1 is associated with lipid metabolism. Notably, confirmation of this theory in GC cells and tissues using qRT-PCR demonstrated that only FASN expression is correlated to MACC1. Therefore, we focused on FASN and MACC1 in the following experiment.

FASN is a key enzyme in lipid metabolism, whose expression has great potential in helping to understand lipidmetabolism disorders $(25,26)$. In the present study, we generally clarified the relationship between FASN and MACC1 in vivo and in vitro. We first confirmed that MACC1 was positively associated with FASN both at the mRNA and protein levels. More importantly, we found that MACC1 could regulate the expression and enzyme activity of FASN, while FASN inhibition could not alter the expression of MACC1. This successfully confirmed that MACC1 was the upstream regulator of FASN.

As aforementioned, chemoresistance is the main cause for the poor prognosis in GC (27). In the present study, we found that MACC1 overexpression was correlated with a poorer prognosis in GC patients who received oxaliplatin treatment, highly indicating that MACC1 works as a druggable gene and contributes to oxaliplatin resistance in GC. In fact, this is not the first time MACC1 has been found to play a role in drug resistance. It was reported that MACC1 overexpression induced cisplatinum resistance in ovarian cancer $(28,29)$. Therefore, it is possible and reasonable that MACC1 contributes to oxaliplatin resistance in GC. We further explored the detailed mechanism and found that FASN participated in MACC1-induced oxaliplatin resistance. 
FASN is also considered to be a druggable gene, and FASN overexpression is correlated with tumor drug-resistance, which can partially explain the relationship between FASN and poor prognosis. In the present study, an siRNA targeted to FASN or semi-synthetic inhibitor C75, were employed to clarify the role of FASN in MACC1-induced oxaliplatin resistance. We found that both siRNA and C75 significantly reversed the downregulation of chemosensitivity to oxaliplatin induced by MACC1 overexpression, greatly indicating that FASN was the key link in MACC1-induced oxaliplatin resistance and inhibition of FASN may be a therapeutic strategy for oxaliplatin resistance.

A previous study utilized gene expression profile microarray analysis and found that pro-apoptotic genes and proteins were increased in FASN-knockout breast cancer cells (30). It has been considered that knockout of FASN plays a dominant role in the upregulation of ceramide levels and consequently upregulates the expression of these pro-apoptotic genes. However, the concrete underlying mechanism remains unclear. Liu et al found that FASN overexpression in breast cancer could suppress doxorubicin-induced cell apoptosis and caspase- 8 activation (10). The present study contends that apoptosis or DNA-damage are among the main mechanisms of FASN-mediated drug-resistance. However, whether MACC1-facilitated oxaliplatin resistance of GC is associated with those factors requires further investigation. Undeniably, MACC1 had an impact on GC chemosensitivity to oxaliplatin through interaction with FASN.

In conclusion, our study explored lipometabolism, confirmed the relationship between MACC 1 and the key enzyme of lipogenesis, FASN, and further elucidated the role of MACC1 in cancer metabolism. In combination with clinical data, we revealed for the first time the role of MACC1 in the chemosensitivity of oxaliplatin, confirmed that silencing of MACC1 improved the chemosensitivity of GC cells to oxaliplatin by downregulation of FASN, and highly suggest that targeting FASN may be a new therapeutic strategy for the reversal of oxaliplatin-resistance.

\section{Acknowledgements}

The present study was funded by grants from the National Natural Science Foundation of China (81502116 to L.S.), grants from the Scientific and Technological Projects of Nanshan District, Shenzhen (2015011 to J.-M.D.), the Team Program of Natural Science Foundation of Guangdong Province, China (no. S2011030003134 to W.-J.L.), the Team Program of Natural Science Foundation of Guangdong Province, China (2015A030310085 to L.S.), and the Key Clinical Specialty Discipline Construction Program of China (to the Department of Oncology, Nanfang Hospital).

\section{References}

1. Jemal A, Bray F, Center MM, Ferlay J, Ward E and Forman D: Global cancer statistics. CA Cancer J Clin 61: 69-90, 2011.

2. Peinert S, Grothe W, Stein A, Müller LP, Ruessel J, Voigt W, Schmoll HJ and Arnold D: Safety and efficacy of weekly 5-fluorouracil/folinic acid/oxaliplatin/irinotecan in the first-line treatment of gastrointestinal cancer. Ther Adv Med Oncol 2: 161-174, 2010.
3. Stein U, Walther W, Arlt F, Schwabe H, Smith J, Fichtner I, Birchmeier W and Schlag PM: MACC1, a newly identified key regulator of HGF-MET signaling, predicts colon cancer metastasis. Nat Med 15: 59-67, 2009.

4. Lin L, Huang H, Liao W, Ma H, Liu J, Wang L, Huang N, Liao Y and Liao W: MACC1 supports human gastric cancer growth under metabolic stress by enhancing the Warburg effect. Oncogene 34: 2700-2710, 2015.

5. Wang L, Wu Y, Lin L, Liu P, Huang H, Liao W, Zheng D, Zuo Q, Sun L, Huang N, et al: Metastasis-associated in colon cancer-1 upregulation predicts a poor prognosis of gastric cancer, and promotes tumor cell proliferation and invasion. Int J Cancer 133: 1419-1430, 2013.

6. Djiogue S, Nwabo Kamdje AH, Vecchio L, Kipanyula MJ, Farahna M, Aldebasi Y and Seke Etet PF: Insulin resistance and cancer: The role of insulin and insulin-like growth factors. Endocr Relat Cancer 20: R1-R17, 2013.

7. Hiller K and Metallo CM: Profiling metabolic networks to study cancer metabolism. Curr Opin Biotechnol 24: 60-68, 2013.

8. Tamada M, Nagano O, Tateyama S, Ohmura M, Yae T, Ishimoto T, Sugihara E, Onishi N, Yamamoto T, Yanagawa H, et al: Modulation of glucose metabolism by CD44 contributes to antioxidant status and drug resistance in cancer cells. Cancer Res 72: 1438-1448, 2012.

9. Zhang Y and Yang JM: Altered energy metabolism in cancer: A unique opportunity for therapeutic intervention. Cancer Biol Ther 14: 81-89, 2013.

10. Liu H, Wu X, Dong Z, Luo Z, Zhao Z, Xu Y and Zhang JT: Fatty acid synthase causes drug resistance by inhibiting TNF- $\alpha$ and ceramide production. J Lipid Res 54: 776-785, 2013.

11. Yang Y, Liu H, Li Z, Zhao Z, Yip-Schneider M, Fan Q, Schmidt CM, Chiorean EG, Xie J, Cheng L, et al: Role of fatty acid synthase in gemcitabine and radiation resistance of pancreatic cancers. Int J Biochem Mol Biol 2: 89-98, 2011.

12. Wangpaichitr M, Sullivan EJ, Theodoropoulos G, Wu C, You M, Feun LG, Lampidis TJ, Kuo MT and Savaraj N: The relationship of thioredoxin-1 and cisplatin resistance: Its impact on ROS and oxidative metabolism in lung cancer cells. Mol Cancer Ther 11: 604-615, 2012.

13. Menendez JA and Lupu R: Fatty acid synthase and the lipogenic phenotype in cancer pathogenesis. Nat Rev Cancer 7: 763-777, 2007.

14. Kuhajda FP: Fatty-acid synthase and human cancer: New perspectives on its role in tumor biology. Nutrition 16: 202-208, 2000.

15. Xiang HG, Hao J, Zhang WJ, Lu WJ, Dong P, Liu YB and Chen L: Expression of fatty acid synthase negatively correlates with PTEN and predicts peritoneal dissemination of human gastric cancer. Asian Pac J Cancer Prev 16: 6851-6855, 2015.

16. Hashimoto T, Kusakabe T, Sugino T, Fukuda T, Watanabe K, Sato Y, Nashimoto A, Honma K, Kimura H, Fujii H, et al: Expression of heart-type fatty acid-binding protein in human gastric carcinoma and its association with tumor aggressiveness, metastasis and poor prognosis. Pathobiology 71 : 267-273, 2004

17. Duan J, Sun L, Huang H, Wu Z, Wang L and Liao W: Overexpression of fatty acid synthase predicts a poor prognosis for human gastric cancer. Mol Med Rep 13: 3027-3035, 2016.

18. Shiragami R, Murata S, Kosugi C, Tezuka T, Yamazaki M, Hirano A, Yoshimura Y, Suzuki M, Shuto K and Koda K: Enhanced antitumor activity of cerulenin combined with oxaliplatin in human colon cancer cells. Int J Oncol 43: 431-438, 2013.

19. Liu H,Liu Y and Zhang JT: A new mechanism of drug resistance in breast cancer cells: Fatty acid synthase overexpression-mediated palmitate overproduction. Mol Cancer Ther 7: 263-270, 2008.

20. Bauerschlag DO, Maass N, Leonhardt P, Verburg FA, Pecks U, Zeppernick F, Morgenroth A, Mottaghy FM, Tolba R, Meinhold-Heerlein I, et al: Fatty acid synthase overexpression: Target for therapy and reversal of chemoresistance in ovarian cancer. J Transl Med 13: 146, 2015.

21. Warburg O: On the origin of cancer cells. Science 123: 309-314, 1956.

22. Menendez JA: Fine-tuning the lipogenic/lipolytic balance to optimize the metabolic requirements of cancer cell growth: Molecular mechanisms and therapeutic perspectives. Biochim Biophys Acta 1801: 381-391, 2010.

23. Furuta E, Okuda H, Kobayashi A and Watabe K: Metabolic genes in cancer: Their roles in tumor progression and clinical implications. Biochim Biophys Acta 1805: 141-152, 2010. 
24. Sun L, Duan J, Jiang Y, Wang L, Huang N, Lin L, Liao Y and Liao W: Metastasis-associated in colon cancer-1 upregulates vascular endothelial growth factor-C/D to promote lymphangiogenesis in human gastric cancer. Cancer Lett 357: 242-253, 2015.

25. Hopperton KE, Duncan RE, Bazinet RP and Archer MC: Fatty acid synthase plays a role in cancer metabolism beyond providing fatty acids for phospholipid synthesis or sustaining elevations in glycolytic activity. Exp Cell Res 320: 302-310, 2014.

26. Chang L, Wu P, Senthilkumar R, Tian X, Liu H, Shen X, Tao Z and Huang P: Loss of fatty acid synthase suppresses the malignant phenotype of colorectal cancer cells by down-regulating energy metabolism and mTOR signaling pathway. J Cancer Res Clin Oncol 142: 59-72, 2016.

27. Zhang D and Fan D: New insights into the mechanisms of gastric cancer multidrug resistance and future perspectives. Future Oncol 6: 527-537, 2010.
28. Chen ZM, Shi HR,Li X, Deng YX and Zhang RT: Downregulation of MACC1 expression enhances cisplatin sensitivity in SKOV-3/DDP cells. Genet Mol Res 14: 17134-17144, 2015.

29. Zhang R, Shi H, Ren F, Li X, Zhang M, Feng W and Jia Y: Knockdown of MACC1 expression increases cisplatin sensitivity in cisplatin-resistant epithelial ovarian cancer cells. Oncol Rep 35: 2466-2472, 2016.

30. Hilvo M, Denkert C, Lehtinen L, Müller B, Brockmöller S, Seppänen-Laakso T, Budczies J, Bucher E, Yetukuri L, Castillo $\mathrm{S}$, et al: Novel theranostic opportunities offered by characterization of altered membrane lipid metabolism in breast cancer progression. Cancer Res 71: 3236-3245, 2011. 